\title{
Origem da matéria orgânica sedimentar no delta-estuarino do Rio São Francisco, AL/SE - Brasil
}

\author{
Elisamara Sabadini Santos ${ }^{1 *}$ \\ Tim Jennerjahn ${ }^{2}$ \\ Thomas Leipe ${ }^{3}$ \\ Paulo Ricardo Petter Medeiros ${ }^{4}$ \\ Weber Friederichs Landim de Souza ${ }^{5}$ \\ Bastiaan Adriaan Knoppers ${ }^{1}$
}

'Universidade Federal Fluminense (UFF) - Niteroi (RJ), Brasil.

2Leibniz Institut für Marine Tropenökologie -

Bremen, Alemanha.

${ }^{3}$ Leibniz Institut für Ostseeforschung

Warnemünde - Rostock, Alemanha.

${ }^{4}$ Universidade Federal de Alagoas (UFAL) -

Maceió (AL), Brasil.

${ }^{5}$ Instituto Nacional de Tecnologia (INT) -

Rio de Janeiro (RJ), Brasil.

*Autor correspondente: esabadini@geoq.uff.br

\section{Resumo}

O Rio São Francisco (RSF) possui uma cascata de barragens, construídas entre 1975 e 1994, que reduziu drasticamente a vazão e o aporte de matéria para a zona costeira. Este trabalho caracterizou as contribuições relativas das fontes de matéria orgânica sedimentar no estuário do RSF em 15 amostras superficiais. A distribuição granulométrica espacial, com teor de finos predominantemente inferiores a $70 \%$, revelou o caráter dinâmico da região. Ambientes mais deposicionais foram identificados apenas no canal secundário do estuário, que atravessa um manguezal, na margem sul, e em uma pequena enseada fluvial. A quantidade de matéria orgânica aumentou com o teor de sedimentos finos $(<63 \mu \mathrm{m})$, sendo os maiores valores encontrados nas regiões mais deposicionais. Os baixos valores de carbono orgânico $(0,02-6,74 \%)$, nitrogênio total $(0,00-0,33 \%)$, fósforo total $(18-539 \mu \mathrm{g} / \mathrm{g})$ e razão molar C/N (11-45) nos sedimentos refletem a oligotrofia do sistema. Os valores de $\delta^{13} \mathrm{C}$ e o $\delta^{15} \mathrm{~N}$ nos sedimentos variaram, respectivamente, de $-28,24$ e $2,20 \%$ na região do mangue a $-20,30$ e $8,21 \%$ em uma pequena enseada fluvial, valores distribuídos entre os sinais isotópicos das espécies vegetais Elodea sp. (-15,77 e 4,10\%), Montrichadia linífera (-26,93 e 5,48\%o) e Rhizophora mangle (-28,21 e 3,05\% ), de ampla ocorrência na área de estudo. A assinatura isotópica de algumas amostras indica ainda contribuição de uma quarta fonte de matéria orgânica significativa na região, possivelmente o fitoplâncton. A lignina confirmou a importância das três espécies vegetais analisadas, evidenciando a contribuição preferencial da origem flúvio-terrestres e indicando que a matéria orgânica sedimentada foi pouco degradada.

Palavras-chave: sedimento, matéria orgânica, isótopo estável, lignina.

\section{Abstract}

The São Francisco River (RSF) has a cascade of dams, constructed from 1975 to 1994, along its middle and middle-lower basin, which reduced the discharge, the input of dissolved and the particulate matter to the coastal zone. This study characterized the relative contributions of terrestrial and marine sources of organic matter for the total sediments of the RSF estuary, in 15 surface samples. The spatial grain size variability, in which fine-grained sediments are less than $70 \%$ of the total, indicates highly dynamic characteristic of the region. Depositional areas were identified only in the enclosed part of the mangrove and in a fluvial embayment. The amount of organic matter increased as the fine-grained sediment fraction $(<63 \mu \mathrm{m})$ increases, the instead of which highest values were observed in enclosed regions. The low values of organic carbon $(0.02-6.64 \%)$, total nitrogen (0.00-0.33\%), total phosphorus $(18-539 \mu \mathrm{g} / \mathrm{g})$ and C/N molar 
ratio (11-45) in the sediments reflect an oligotrophic environment. The $\delta 13 \mathrm{C}$ and $\delta 15 \mathrm{~N}$ in the sediments vary, respectively, from -28.24 and $2.20 \%$ in the mangrove area to -20.30 and $8.21 \%$ in the small fluvial embayment. These values reflect strong influence of isotopic signature of the three important plant species in the region: Elodea sp. (-15.77 and 4.10\%o), Montrichadia linifera Schott (-26.93 and 5.48\%o) and Rhizophora mangle (-28.21 and 3.05\%o). The isotopic signature of some samples also indicates a contribution of a fourth significant source of organic matter to the region, possibly the phytoplankton. Lignin confirmed the importance of the three plant species analyzed, indicating the main contribution of riverine-terrestrial sources and evidencing that the sedimentary organic matter was little degraded.

Keywords: sediment, organic matter, stable isotope, lignin.

\section{INTRODUÇÃO}

A zona costeira representa uma das áreas de transição da interface continente-oceano, abrangendo ambientes como estuários, baías, lagoas, marismas e manguezais, e corresponde a um dos elos que controlam o fluxo e o destino de água e matéria do ciclo global (Ver et al. 1999, Mantoura et al. 1991). Em particular, os estuários têm a característica de serem ambientes dinâmicos, resultado da interação e variabilidade temporal entre o aporte fluvial e a ação da maré e de ondas (Pritchard 1952). Embora contribuam no seu conjunto com uma parcela de $16 \%$ da produção primária da margem oceânica e $4 \%$ da produção primária marinha oceânica global, atuam como principais reatores na transformação de matéria biogênica na interface terra-mar (Billen et al. 1991, Knoppers et al. 2006). Além disso, os fluxos de água e matéria dissolvida e particulada para a zona costeira vêm sendo significativamente alterados por intervenções antrópicas no uso e manejo das bacias de drenagem, inclusive no Brasil, pela construção de barragens fluviais para a geração de energia elétrica e irrigação (Tundisi et al. 1998, ANA/GERCO/PNUMA/OEA 2003).

O Rio São Francisco (RSF) tem sua nascente em Minas Gerais e desembocadura no limite de Alagoas e Sergipe; possui, ao longo de seu médio-baixo curso, uma cascata de barragens, que representa um exemplo de destaque com alterações na região estuarina (Bessa \& Paredes 1990, Marques et al. 2004, Knoppers et al. 2006, Medeiros et al. 2007 e 2011, Sabadini-Santos 2009). A cascata de barragens ao longo do RSF, que controla $98 \%$ da bacia de drenagem, reduziu drasticamente a vazão, a carga fluvial de matéria

\section{MATERIAL E MÉTODOS}

\subsection{Caracterização da área}

A nascente do RSF se localiza na Serra da Canastra, no estado de Minas Gerais. O rio percorre no sentido Norte a longa depressão encravada entre o Planalto Atlântico e as Chapadas do Brasil Central até atingir a divisa do estado da Bahia e de Pernambuco, quando, então, passa a se deslocar no sentido Leste. Após percorrer uma extensão de $2.863 \mathrm{~km}$ em suspensão e de nutrientes, e praticamente eliminou o padrão sazonal de descarga de água e materiais associados (Knoppers et al. 2006). Devido à retenção de sólidos em suspensão nos reservatórios, principalmente no de Sobradinho à montante da cascata, o rio tornou-se transparente e oligotrófico (Medeiros et al. 2007, 2011, Knoppers et al. 2006) e diversos lagos e várzeas de suas margens deixaram de ser sazonalmente inundados e fertilizados, alterando o funcionamento físico-biogeoquímico, o rendimento pesqueiro e, em muitos casos, levando-os à secura total. A diminuição do aporte dos sedimentos fluviais alterou o balanço sedimentar do estuário, desencadeou o processo de desbarrancamento das margens (que contribui para o assoreamento de determinados trechos fluviais) e acelerou o processo de erosão costeira (Oliveira 2003, Dominguez 1996, Bittencourt et al. 2007).

Sob o enfoque da geoquímica inorgânica e orgânica dos ambientes sedimentares, o estuário do Rio São Francisco foi somente estudado quanto à composição e ao comportamento de metais majoritários e traços (Sabadini-Santos et al. 2009).

Este trabalho tem o objetivo de identificar as regiões deposicionais remanescentes no estuário do RSF após regularização da vazão fluvial e caracterizar a matéria orgânica sedimentar a nível elementar e isotópico de carbono e nitrogênio e molecular de fenóis de ligninas. Com o conjunto destas ferramentas de marcadores, pretende-se verificar as assinaturas registradas nos sedimentos em comparação às fontes da matéria orgânica alóctone e autóctone, e determinar a origem atual da matéria orgânica sedimentada, após a construção das barragens. de solos e climas diferenciados, desemboca no oceano Atlântico à latitude $10^{\circ} 25^{\prime} \mathrm{S}$ e longitude $36^{\circ} 23^{\prime} \mathrm{W}$, entre os estados de Sergipe e Alagoas (Figura 1).

A bacia de drenagem $\left(\mathrm{A}^{\mathrm{B}}\right)$ possui uma área de $639.219 \mathrm{~km}^{2}$ e é subdividida em 4 setores (CODEVASF 1975, ANA/GERCO/PNUMA/OEA 2003): (1) Alto, desde 
a nascente do rio até ao redor da cidade de Pirapora (MG), com área de $110.696 \mathrm{~km}^{2}$ (17\% da bacia); (2) Médio, com área de $322.140 \mathrm{~km}^{2}$ (50\% da bacia), até a região montante do reservatório de Sobradinho; (3) Submédio, com área de $168.528 \mathrm{~km}^{2}$ (26\% da bacia), incorporando a cascata de barragens até Xingó; e (4) Baixo, desde Xingó até a foz, com área de $36.959 \mathrm{~km}^{2}$ (6\% da bacia; ANA/GERCO/PNUMA/OEA 2003). O Alto RSF apresenta clima tipo Cwa na nascente e Aw com chuvas de verão; o Médio RSF, clima de transição de Aw a BShw; o Submédio RSF, clima semiárido tipo BSh; e o Baixo RSF, clima AS, com chuvas de outono-inverno (Bernardes 1971, Medeiros et al. 2007).

Antes da construção da cascata de barragens, a vazão variava principalmente de acordo com a pulsação sazonal das chuvas no Alto e Médio RSF, com picos entre 8.000 e $18.000 \mathrm{~m}^{3} \mathrm{~s}$ e baixas na primavera de $600 \mathrm{~m}^{3} / \mathrm{s}$. Com a construção das barragens de 1975 até 1994, Sobradinho (1973-1980) localizada a $800 \mathrm{~km}$ adentro da costa e Xingó (1994) a 180 km da costa, a vazão foi definitivamente regularizada em 1995 pela barragem de Sobradinho. Atualmente, no período pós-barragens, a vazão regularizada se manifesta num patamar constante de, em média, $1.850 \mathrm{~m}^{3} / \mathrm{s}$ ( $35 \%$ menor que no período

\subsection{Amostragem}

O delta estuarino do RSF $\left(10^{\circ} 25^{\prime}\right.$ S e $\left.36^{\circ} 23^{\prime} \mathrm{W}\right)$ apresenta atualmente, além do canal central, um canal secundário de escoamento: o canal do Funil (Mauriel 2004). As estações S1, S2, S3 e S4 estão localizadas ao longo do canal fluvial central. Já as S5, S6, S7, S8, S9, S10, S11, S12, S13, S14 foram amostradas ao longo do canal secundário do Funil, cuja entrada se localiza na região estuarina do canal central e percorre um mangue bem desenvolvido, até alcançar o mar $25 \mathrm{~km}$ ao Sul do canal central. A estação $\mathrm{CP}$ se encontra em um depósito lamoso na plataforma continental, adjacente à foz (Figura 1). Ambos os transectos, do canal central e do canal do Funil, representam um gradiente estuarino do sistema. Todas as amostras de sedimento superficial foram coletadas com um amostrador pré-barragens) e o reservatório de Sobradinho com volume de $34 \mathrm{~km}^{3}$ e $350 \mathrm{~km}$ de extensão retém até $95 \%$ da matéria em suspensão carreada dos setores Alto e Médio RSF, empobrecendo, assim, o estuário com matéria em suspensão e nutrientes e alterando o padrão de migração do gradiente estuarino e o balanço hidro-sedimentar, além de incrementar a erosão no rio e na zona costeira (Oliveira 2003, Medeiros et al. 2007 e 2011).

A área do delta-estuarino com os depósitos sedimentares das transgressões/regressões do Pleistoceno Tardio e do Holoceno compreende $800 \mathrm{~km}^{2}$ e o rio transversa a área com $30 \mathrm{~km}$ até a foz (Dominguez 1996). A maré é do tipo semidiurno, enquadra-se na categoria de meso-marés (i.e. $>2 \mathrm{~m}<4 \mathrm{~m}$ ), com faixa de até 2,7 $\mathrm{m}$ na Sizígia. Ventos do NE e E predominam no verão e do SE no inverno. $\mathrm{O}$ regime de alta energia de ondas se manifesta com altura média de $1 \mathrm{~m}$ e frequência de $6 \mathrm{~s}$ (Medeiros et al. 2007, Bittencourt et al. 2007). O conjunto destes fatores acarreta alta dinâmica dos processos físicos no estuário. Entretanto, devido à regularização da vazão, a sua cunha salina somente se prolifera em torno de $10 \mathrm{~km}$ adentro e a influência da maré no rio acima da intrusão salina (i.e. setor rio da maré) alcança em torno de $30 \mathrm{~km}$.

de fundo tipo "Van-Veen". Uma alíquota foi separada e mantida sob refrigeração para análise granulométrica e o restante dos sedimentos foi seco em estufa a $40^{\circ} \mathrm{C}$ até peso constante, triturado e homogeneizado para posterior análise dos compostos químicos biogênicos.

Três espécies vegetais foram coletadas em função de sua ampla ocorrência (Santos 2001) para análises de composição elementar, isotópica e molecular (ligninas) após lavadas em água corrente, secas a $40^{\circ} \mathrm{C}$ na estufa, trituradas e maceradas. Como espécie mais representativa do manguezal bem desenvolvido ao longo de todo Canal do Funil, coletaram-se amostras do vegetal superior Rhizophora mangle. A Montrichadia linifera (aninga) é encontrada ao longo de praticamente toda a
Figura 1

Mapa da área de estudo com a localização das estações de coleta de sedimento superficial no delta-estuarino do Rio São Francisco, Nordeste do Brasil.

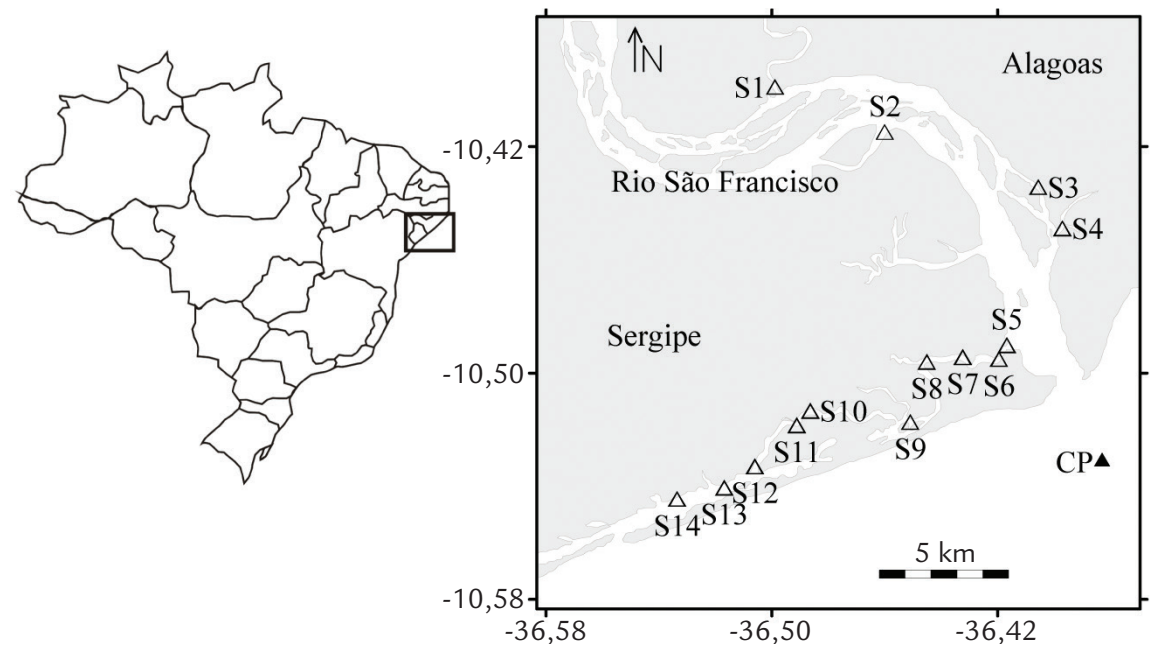


margem do Baixo São Francisco, até a região estuarina, e caracteriza-se por ser uma planta aquática, de até $5 \mathrm{~m}$ de altura e $20 \mathrm{~cm}$ de diâmetro, caule ereto e fusiforme, folhas grandes sagitadas, com flores em espiga e fruto bacilar grande contendo numerosas sementes (Santos 2001). A macroalga Elodea sp. vem se proliferando ao longo do

\subsection{Métodos analíticos e estatísticos}

A granulometria sedimentar foi determinada por meio de um analisador de partículas com laser ótico CILAS 1180 L no Instituto de Estudos do Mar Báltico - Warnemünde (IOW), Alemanha. Alíquotas secas das amostras foram tratadas com $\mathrm{H}_{2} \mathrm{O}_{2}$ para remoção da matéria orgânica. $\mathrm{Na}$ sequência houve banho de ultrassom e adição de polifosfafto de potássio para evitar recoagulação. A classificação granulométrica adotada foi a proposta por Folk e Ward (1957). $\mathrm{O}$ carbono orgânico e o nitrogênio total foram determinados no Instituto de Ecologia Tropical Marinha - Bremen (ZMT), Alemanha, por combustão a alta temperatura no analisador elementar Carlo Erba NA 2100 após acidificação delas para remoção de carbonatos. Os isótopos estáveis de carbono $\left(\delta^{13} \mathrm{C}\right)$ e nitrogênio $\left(\delta^{15} \mathrm{~N}\right)$ foram determinados com espectrômetro de massa de razão isotópica gasosa Tipo Finnigan Delta Plus após combustão a alta temperatura em um analisador elementar Carlo Erba Flash 1112EA (padrões V-PDB e AIR). O fósforo orgânico foi determinado de acordo com o método de ignição de Aspila et al. (1976), obtido indiretamente pela diferença entre o fósforo total e o fósforo inorgânico, e a concentração de fósforo total e inorgânico foi determinada por espectrofotometria de absorção molecular (Koroleff 1976).

A contribuição relativa e a composição da matéria orgânica de origem terrestre foram investigadas por meio da análise dos produtos de oxidação dos fenóis de lignina leito do rio e prejudicando a pesca (Santos 2001). Esta proliferação é consequência da ausência de enchentes para carregá-las e da drástica diminuição do material particulado em suspensão, o que aumenta a penetração da luz na coluna de água e propicia melhores condições de crescimento para os produtores primários.

(Hedges \& Ertel 1982, Hedges \& Mann 1979). A análise de lignina efetuou-se após oxidação com $\mathrm{CuO}$ (Hedges \& Ertel 1982, Kögel \& Bochter 1985). O procedimento exato foi detalhado por Miltner e Emeis (2001) e a recuperação da lignina determinada por padrão interno, variando de 50 a $110 \%$, com média de $75 \%$. Amostras representativas foram analisadas em duplicata para determinar a reprodutibilidade do método, resultando em coeficientes de variação menores que $25 \%$, anto para o somatório dos produtos de oxidação da lignina quanto para os índices. Na Tabela 1 encontram-se as definições dos grupos de compostos da oxidação da lignina bem como dos índices entre os fenóis de lignina e de degradação utilizados neste trabalho.

As análises estatísticas foram realizadas no programa STATISTICA 5.1 (Copyright $^{\oplus}$ 1984-97, StatSoft, Inc.), sendo a normalidade dos parâmetros testada por meio do teste Shapiro-Wilk $(\mathrm{p}<0,05)$, cuja conclusão foi que a maioria dos parâmetros não teve distribuição normal. Desta forma, empregou-se a correlação não paramétrica de Spearman, que opera com ranking das medidas para cada variável (Zar 1982). A análise multiparamétrica foi executada com a matriz transformada pelo método ranging $\left(\mathrm{x}-\mathrm{x}^{\text {mín/ }} \mathrm{x}^{\text {máx }}-\mathrm{x}^{\text {mín }}\right)$, o qual, segundo Milligan 1988, é superior à transformação escore $\mathrm{Z}\left(\mathrm{x}-\right.$ média $^{\mathrm{x}} /$ desvio-padrão $\left.{ }^{\mathrm{x}}\right)$. Realizou-se a análise de cluster mediante método de agrupamento Ward, com distância de ligação Manhattan.

\begin{tabular}{lr}
\hline Parâmetro & Definição \\
\hline $\mathrm{V}$ & Somatório das formas ácida, aldeídica e cetônica da vanilina \\
\hline $\mathrm{S}$ & Somatório das formas ácida, aldeídica e cetônica do siringil \\
\hline $\mathrm{C}$ & Somatório dos ácidos p-coumárico e ferúlico, caracterizando o grupo cinamil \\
\hline $\mathrm{P}$ & Somatório das formas ácida, aldeídica e cetônica do grupo p-hidroxibenzóico \\
\hline$(\mathrm{ac} / \mathrm{al}) \mathrm{v}$ & Razão molar de ácido vanílico e vanilina \\
\hline$(\mathrm{ac} / \mathrm{al}) \mathrm{s}$ & Razão molar de ácido siríngico e siringaldeído \\
\hline
\end{tabular}

Tabela 1

Lista de abreviações dos compostos

fenólicos oxidados da lignina.

\section{RESULTADOS}

\subsection{Granulometria}

A granulometria dos sedimentos superficiais do estuário do RSF apresentou heterogeneidade espacial marcante (Tabela 2), reflexo das diferentes condições hidrodinâmicas atuantes no Canal Central e no Canal Secundário. Não houve predominância de sedimentos finos $(<63 \mu \mathrm{m})$, salvo algumas estações que apresentaram $70 \%$ ou mais na enseada de domínio fluvial (estação S2), na porção central com manguezais do canal secundário (estações S9 e S11) e no pro-delta (estação CP). Menores teores de sedimentos finos foram observados nas estações próximas à foz (S4, S6 e S13). 


\subsection{Composição biogênica elementar (C, N e P) e dos isótopos estáveis $\left(\delta^{13} C\right.$ e $\left.\delta^{15} N\right)$}

Em geral, os sedimentos superficiais apresentaram baixos teores de matéria orgânica, exceto em alguns sítios enclausurados. Os valores de carbono orgânico $(0,02-6,74 \%)$, nitrogênio total $(0,00-0,33 \%)$ e de fósforo total $(18-$ $539 \mu \mathrm{g} / \mathrm{g}$ ) nos sedimentos refletiram, em geral, a oligotrofia do sistema. A estação $S 2$, no canal central, e as estações $S 7$, S8, S11 e S14, no secundário, apresentaram concentrações de carbono orgânico $\left(\mathrm{C}_{\mathrm{org}}\right)$ superiores a $1 \%$ (Tabela 2$)$, das quais apenas as estações S2 e S11 tiveram também elevado teor de sedimentos finos. As maiores concentrações de nitrogênio total $\left(\mathrm{N}_{\text {total }}\right)$ e fósforo orgânico $\left(\mathrm{P}_{\mathrm{org}}\right)$ ocorreram na estação S2, com elevado teor de sedimentos finos. Já as menores concentrações de $\mathrm{C}_{\text {org }}, \mathrm{N}_{\text {total }}$ e $\mathrm{P}_{\text {org }}$ foram registradas na estação $S 4$, próxima à foz, e nas estações $S 6$ e $S 10$, no canal secundário, todas com alto teor de areia. Tanto o $\mathrm{N}_{\text {total }}(\mathrm{r}=0,70)$ quanto $\mathrm{P}_{\text {org }}(\mathrm{r}=0,67)$ apresentaram correlações significativas $(\mathrm{p}<0,01 ; \mathrm{n}=15)$ com teor de sedimentos finos.

Apenas ao avaliarmos a distribuição das razões elementares $\mathrm{C} / \mathrm{N}$ e $\mathrm{C} / \mathrm{P}$ e da razão isotópica do carbono $\left(\delta^{13} \mathrm{C}\right)$ evidenciamos uma distinção entre canal central e canal secundário, sendo que o último apresenta os valores mais empobrecidos em $\mathrm{N}, \mathrm{P} \mathrm{e}^{13} \mathrm{C}$ (Tabela 2). A razão $\mathrm{C} / \mathrm{N}$ indicou correlação significativa com a razão $\mathrm{C} / \mathrm{P}(\mathrm{r}=0,77$; $\mathrm{p}<0,01 ; \mathrm{n}=15)$ e com o $\delta^{13} \mathrm{C}(\mathrm{r}=-0,68 ; \mathrm{p}<0,01 ; \mathrm{n}=15)$. Em geral, o $\delta^{15} \mathrm{~N}$ também acompanhou o padrão descrito para

\subsection{Composição dos marcadores de ligninas}

A contribuição da fonte terrestre para a matéria orgânica sedimentada pode ser estimada pelo uso de produtos da oxidação da lignina como marcadores moleculares, incluindo o Vanilil (V), Siringil (S) e Cinamil (C). O somatório V+S+C é proporcional ao conteúdo de lignina total, sendo, assim, um indicador da contribuição terrestre para a matéria orgânica total (Hegdes \& Mann 1979). Os produtos de oxidação da o $\delta^{13} \mathrm{C}$, corroborado com correlação significativa $(\mathrm{r}=0,52$; $\mathrm{p}<0,05 ; \mathrm{n}=15)$.

Embora nenhuma medida de biomassa tenha sido executada em campo, os sinais isotópicos dos sedimentos superficiais refletiram o sinal isotópico das espécies vegetais consideradas de ampla ocorrência na área estudada. A estação S2, na pequena enseada localizada no canal central, apresentou o maior valor de $\delta^{13} \mathrm{C}$ encontrado nos sedimentos superficiais, onde a espécie Elodea sp. é dominante $\left(\delta^{13} \mathrm{C}=-15,77 \%\right)$. A macroalga Elodea sp., de ampla ocorrência no leito do canal central, registrou os menores valores de razão $\mathrm{C} / \mathrm{N}(25,13)$. A aninga, Montrichadia linifera, abundante nas margens do canal central, também apresentou baixa razão molar $\mathrm{C} / \mathrm{N}$ $(25,22)$ e o mais pesado $\delta^{15} \mathrm{~N}(5,48 \%)$, influenciando a estação S3 do canal central, por exemplo. A razão C/N mais elevada e o $\delta^{13} \mathrm{C}$ mais leve da estação S11, localizada no meio do canal do Funil, estão relacionados à abundante ocorrência da espécie vegetal da Rhizophora mangle, que indicou elevada razão $\mathrm{C} / \mathrm{N}(50,89)$ e o $\delta^{13} \mathrm{C}$ mais leve $(-28,21 \%)$.

A estação $\mathrm{CP}$ registrou concentrações de $\mathrm{C}_{\mathrm{org}}, \mathrm{N}_{\text {total }}, \mathrm{P}_{\mathrm{org}}$ e suas respectivas razões elementares muito semelhantes às medianas do canal central, indicando a importância da fonte fluvial para esses sedimentos (Tabela 2). Já o $\delta^{13} \mathrm{C}$ um pouco mais pesado $(-22,35 \%)$ sugere mistura de matéria orgânica marinha com a de origem fluvio-terrestre.

lignina variaram de 361 a $1.649 \mathrm{mg} / \mathrm{g}$ de sedimento seco, sendo os compostos dos grupos $\mathrm{V}$ e $\mathrm{S}$ mais abundantes que os de C (Tabela 3).

A estação arenosa S4 (próxima à foz no canal central) apresentou as máximas concentrações de V, S e do somatório $(\mathrm{V}+\mathrm{S}+\mathrm{C})$. As concentrações mínimas destes parâmetros ocorreram na estação S2 (Tabela 3), enseada no canal central

Tabela 2

Teor de sedimentos finos (<63 mm), concentrações de C, N e P orgânicos, razões elementares $(\mathrm{C} / \mathrm{N}$ e $\mathrm{C} / \mathrm{P})$ e isotópicas $(\delta 13 \mathrm{CV}$ -

PDB e $\delta 15$ NAIR) nos sedimentos superficiais do estuário do Rio São Francisco.

\begin{tabular}{|c|c|c|c|c|c|c|c|c|c|}
\hline Estações & & $\begin{array}{c}\text { Teor de } \\
\text { finos (\%) }\end{array}$ & $\begin{array}{l}C_{\text {org }} \\
(\%)\end{array}$ & $\begin{array}{l}N_{\text {total }} \\
(\%)\end{array}$ & $\begin{array}{l}P_{\text {org }} \\
(\%)\end{array}$ & $\begin{array}{c}\mathrm{C} / \mathrm{N} \\
\text { (molar) }\end{array}$ & $\begin{array}{c}\mathrm{C} / \mathrm{P} \\
\text { (molar) }\end{array}$ & $\begin{array}{l}\delta^{13} \mathrm{C} \\
(\% \circ)\end{array}$ & $\begin{array}{l}\delta^{15} \mathrm{~N} \\
(\% \circ)\end{array}$ \\
\hline S1 & \multirow{4}{*}{$\begin{array}{c}\text { Canal } \\
\text { Central }\end{array}$} & 64,55 & 0,93 & 0,07 & 0,011 & 15 & 223 & $-26,49$ & 4,05 \\
\hline S2 & & 99,87 & 2,01 & 0,21 & 0,020 & 11 & 266 & $-20,30$ & 6,41 \\
\hline S3 & & 66,14 & 0,60 & 0,05 & 0,006 & 14 & 244 & $-24,03$ & 6,77 \\
\hline S4 & & 19,14 & 0,39 & 0,02 & 0,003 & 21 & 289 & $-25,91$ & 5,93 \\
\hline S5 & \multirow{10}{*}{$\begin{array}{l}\text { Canal do } \\
\text { funil }\end{array}$} & 41,14 & 0,82 & 0,04 & 0,005 & 26 & 410 & $-25,16$ & 5,80 \\
\hline S6 & & 27,27 & 0,44 & 0,02 & 0,003 & 21 & 372 & $-25,10$ & 5,70 \\
\hline S7 & & 45,71 & 2,51 & 0,09 & 0,002 & 31 & 3.444 & $-26,08$ & 4,37 \\
\hline S8 & & 30,40 & 1,23 & 0,04 & 0,002 & 34 & 1.314 & $-25,56$ & 5,20 \\
\hline S9 & & 99,27 & 0,93 & 0,06 & 0,012 & 19 & 203 & $-23,93$ & 4,35 \\
\hline $\mathrm{S} 10$ & & 19,96 & 0,36 & 0,02 & 0,004 & 18 & 251 & $-25,50$ & 3,54 \\
\hline $\mathrm{S} 11$ & & 87,17 & 1,66 & 0,04 & 0,007 & 44 & 636 & $-26,67$ & 2,47 \\
\hline $\mathrm{S} 12$ & & 26,94 & 0,68 & 0,03 & 0,003 & 27 & 532 & $-26,09$ & 3,85 \\
\hline $\mathrm{S} 13$ & & 21,89 & 0,99 & 0,05 & 0,004 & 21 & 688 & $-24,93$ & 4,46 \\
\hline S14 & & 33,04 & 1,18 & 0,08 & 0,008 & 18 & 393 & $-23,70$ & 4,11 \\
\hline$C P$ & Pró-delta & 98,67 & 0,63 & 0,06 & 0,004 & 12 & 258 & $-22,35$ & 5,64 \\
\hline
\end{tabular}

$\mathrm{C}_{\text {org }}$ : carbono orgânico; $\mathrm{N}_{\text {total }}$ : nitrogênio total; $\mathrm{P}_{\text {org }}$ : fósforo orgânico; $\mathrm{C} / \mathrm{N}$ : razão carbono/ nitrogênio; C/P: razão carbono/fósforo; $\delta 13 \mathrm{C}$ : razão isotópica do carbono; $\delta 15 \mathrm{~N}$ : razão isotópica do nitrogênio. 
com elevados teores de sedimentos finos. A estação CP no pró-delta indicou concentrações de fenóis da lignina pouco inferiores às amostras do canal central. A razão (ac/al)v apresentou-se praticamente homogênea entre todas as analisadas e sempre menor que 0,4 (Tabela 3). O maior valor da razão (ac/al)v ocorreu na estação $S 2$, onde também ocorreu maior razão $\mathrm{P} / \mathrm{V}+\mathrm{S}$, em que $\mathrm{P}$ é o somatório das formas ácida, aldeídica e cetônica do grupo p-hidroxibenzóico.

A Rhizophora mangle, presente em abundância no canal secundário, registrou as maiores concentrações dos grupos $\mathrm{V}$, $S$ e da razão $S / V$ entre as espécies amostradas (Tabela 3). Em geral, todas as espécies apresentaram baixas concentrações de fenóis dos grupos $\mathrm{P}$ e C, o que provavelmente refletiu a relativa proporção menor desses grupos no montante total de ligninas nos sedimentos superficiais. Todavia, os mesmos estiveram presentes em maior proporção na macroalga Elodea sp., o que é característico de vegetais inferiores, evidenciado pela elevada razão $\mathrm{P} /(\mathrm{V}+\mathrm{S})$ (Tabela 3).

As maiores concentrações de fenóis do grupo $\mathrm{C}$ e da razão C/V ocorreram na estação S1 do canal central (Tabela 3) e a concentração máxima do grupo $P$, na estação $S 4$, próxima à foz do canal central. Entretanto, a maior proporção relativa do grupo $\mathrm{P}$, visualizada pela razão $\mathrm{P} /(\mathrm{V}+\mathrm{S})$, foi encontrada na estação S2 (Tabela 3). Este fato está ligado à influência da macroalga Elodea sp., espécie abundante nesta estação e com alta razão $\mathrm{P} /(\mathrm{V}+\mathrm{S})$.

\begin{tabular}{lcccccccccccc}
\hline $\boldsymbol{\mu g} / \mathbf{g}$ & S1 & S2 & S3 & S4 & S6 & S9 & S11 & S14 & CP & $\begin{array}{c}\text { Elodea } \\
\text { sp. }\end{array}$ & $\begin{array}{c}\text { Montrichardia } \\
\text { linifera }\end{array}$ & $\begin{array}{c}\text { Rhizophora } \\
\text { mangle }\end{array}$ \\
\hline $\mathrm{P}$ & 121 & 240 & 77 & 66 & 73 & 76 & 146 & 89 & 57 & 3043 & 8514 & 1698 \\
\hline $\mathrm{V}$ & 388 & 216 & 179 & 370 & 258 & 323 & 615 & 613 & 191 & 382 & 22348 & 27363 \\
\hline $\mathrm{S}$ & 395 & 224 & 171 & 552 & 281 & 458 & 964 & 1011 & 182 & 420 & 13926 & 101202 \\
\hline $\mathrm{C}$ & 35 & 12 & 11 & 8 & 8 & 11 & 17 & 25 & 8 & 46 & 599 & 73 \\
\hline $\mathrm{V}+\mathrm{S}+\mathrm{C}$ & 818 & 452 & 361 & 929 & 548 & 792 & 1596 & 1649 & 380 & 847 & 36873 & 128638 \\
\hline $\mathrm{S} / \mathrm{V}$ & 1,02 & 1,04 & 0,95 & 1,49 & 1,09 & 1,42 & 1,57 & 1,65 & 0,95 & 1,10 & 1,08 & 1,12 \\
\hline $\mathrm{C} / \mathrm{V}$ & 0,09 & 0,06 & 0,06 & 0,02 & 0,03 & 0,03 & 0,03 & 0,04 & 0,04 & 0,12 & 0,83 & 0,45 \\
\hline $\mathrm{P} /(\mathrm{V}+\mathrm{S})$ & 0,15 & 0,55 & 0,22 & 0,07 & 0,13 & 0,10 & 0,09 & 0,05 & 0,15 & 3,80 & 0,23 & 0,01 \\
\hline (ac/al)v & 0,26 & 0,30 & 0,28 & 0,22 & 0,28 & 0,21 & 0,23 & 0,26 & 0,28 & 0,23 & 0,28 & 0,20 \\
\hline (ac/al)s & 0,13 & 0,13 & 0,12 & 0,11 & 0,13 & 0,09 & 0,11 & 0,12 & 0,12 & 0,08 & 0,12 & 0,13 \\
\hline
\end{tabular}

Tabela 3

Concentrações dos grupos de compostos fenólicos oxidados da lignina e algumas razões entre estes nos sedimentos superficiais do estuário do Rio São Francisco.

P: grupo p-hidroxi; V: grupo Vanilil; S: grupo Siringil; C: grupo Cinamil; (ac/al)v: razão molar de ácido vanílico e vanilina.

\section{DISCUSSÃO}

\subsection{Ambientes hidro-sedimentares}

A granulometria dos sedimentos superficiais analisados e estudos de Oliveira (2003) indicaram a presença de diversos ambientes hidro-sedimentares no estuário do RSF. O primeiro deles é o canal principal perpendicular à costa, com ilhas mais consolidadas nas regiões montante e vegetação das margens submersas dominadas por Montrichadia linifera (Aninga) com altos teores de finos. Abriga também pequenas enseadas com Elodea sp. e sedimentos finos. A área jusante ao mar e além da foz se compõe de bancos deposicionais migratórios de areia. As regiões central e jusante estão sujeitas à resuspensão de materiais do fundo oriundo do próprio rio e do aporte lateral de sedimentos finos das margens em função do bombeamento da maré e da vazão do rio (Medeiros et al. 2007, Knoppers et al. 2006, Sabadini-Santos et al. 2009). Já o canal secundário (canal do Funil) possui três regiões, sendo a conexão com o canal principal caracterizada por sedimentos arenosos e silte. A região central é dominada por manguezais com sedimentos mais finos e a região com conexão ao mar por uma mistura de sedimentos arenosos e silte (Medeiros et al. 2007, 2011, Mauriel 2004, Sabadini-Santos et al. 2009).

A quantidade da matéria orgânica sedimentar no estuário do RSF está, de certa forma, relacionada à granulometria, o que é comumente encontrado em outros ambientes estuarinos (Burone et al. 2003, Baptista Neto et al. 2000). Con- siderando que a distribuição espacial granulométrica revela diferentes condições hidrodinâmicas locais, relacionadas ao aporte de água e sedimento drasticamente modificado pela construção das barragens, a distribuição da matéria orgânica sedimentar também é diversa. Mínimas concentrações de C, $\mathrm{N}$ e $\mathrm{P}$ foram observadas nas estações arenosas próximas à foz (S4, S5, S6, S10, S12 e S13), onde processos erosivos são significativamente acelerados (ANA/GERCO/PNUMA/OEA 2003, Knoppers et al. 2006, Oliveira et al. 2012). A menor competência fluvial em transportar sedimentos, após regularização da vazão fluvial sendo inferior ao patamar das vazões das enchentes do período pré-barragens, permitiu a deposição de partículas finas em suspensão nas estações fluviais mais enclausuradas (S2, S9 e S11).

A estação CP do pró-delta, localizada afora à foz do canal central e dos bancos arenosos migratórios, é composta 99\% de sedimentos finos (Tabela 1). Esta área é de interesse particular uma vez que alimenta, por meio do processo de resuspensão de matéria fina, a pluma de turbidez costeira e, assim, a dispersão de materiais sobre a plataforma continental (Knoppers et al. 2006). Por outro lado, os altos teores de sedimentos finos e as concentrações detectáveis dos produtos de oxidação da lignina encontrados na estação CP também indicam a presença do processo de exportação preferencial de material flúvio-terrestre mais fino para a plataforma continental, que devido à sua 
baixa densidade ultrapassam a primeira fase de retenção de sedimentos arenosos mais densos na desembocadura (Medeiros et al. 2007). Jennerjahn e Ittekkot (1997) constataram que os mínimos valores de matéria orgânica em suspensão (amino ácidos, hexa-aminase carboidratos) na região costeira adjacente à foz do RSF coincidem com o período de maior descarga, uma vez que o material orgânico terrestre oriundo de solos e rios exibe mais baixos valores de carbono e nitrogênio do que a matéria orgânica marinha recém-produzida (Ittekkot \& Zhang 1990, Cowie \& Hedges 1992). Postula-se que o banco lamoso do pró-delta também esteja sofrendo erosão, uma vez que a magnitude da deposição, ou seja, a reposição de material fino proveniente do rio, diminuiu no período pós-barragens, resultado da retenção de materiais nos reservatórios (Knoppers et al. 2006, Medeiros et al. 2007). Mais detalhes sobre a geologia recente, aspectos do nível do mar e erosivos, bem como a dinâmica hidro-sedimentar, relacionados ao processos físicos naturais e influenciados pelas alterações das barragens da costa foram amplamente tratados em Dominguez (1996) e Bittencourt et al. (2007).

\subsection{Composição da matéria biogênica $(C, N$ e $P)$ e isótopos estáveis}

As concentrações de $\mathrm{C}_{\text {org }}, \mathrm{N}_{\text {total }}, \mathrm{P}_{\text {org }}, \mathrm{C} / \mathrm{N}$ e C/P refletem as condições oligotróficas da coluna d'água também identificadas por Mauriel (2004) e Medeiros et al. (2007). O estuário do RSF se apresenta como um ambiente empobrecido de material orgânico, sem aporte significativo de efluentes domésticos e industriais. Os sedimentos dele apresentam teores mínimos de $\mathrm{C}_{\text {org }}, \mathrm{P}_{\text {org }}$ e $\mathrm{C} / \mathrm{N}$ mais pobres que os observados no estuário e na plataforma adjacente à desembocadura, por exemplo, dos rios Mississipi e Amazonas (Ruttemberg \& Goñi 1997). Nota-se o mesmo quando são comparadas as concentrações mínimas deste estudo às concentração de sedimentos de manguezal, de rio e da plataforma continental da costa leste brasileira de 8 a $24^{\circ}$ S (Jennerjahn \& Ittekkot 2002).

A alta correlação entre $\mathrm{C}_{\text {org }}$ e $\mathrm{N}_{\text {total }}(\mathrm{r}=0,73 ; \mathrm{p}<0,01$; $\mathrm{n}=15$ ) é um indício de que as fontes de matéria orgânica estão presentes em proporções constantes ao longo do estuário (Ruttemberg \& Goñi 1997). Existem duas hipóteses para explicar a manutenção da proporção constante de $\mathrm{C} / \mathrm{N}$. A primeira é que fontes terrestre e marinha podem estar sendo eficientemente misturadas antes da deposição final. A segunda, que a matéria orgânica sedimentada já foi alterada durante o seu transporte. Como já indicado acima, a ressuspensão é um processo eficiente que mistura materiais de diversas fontes. Segundo Hedges et al. (1986) o único processo que poderia mascarar a relação entre $\mathrm{C}_{\text {org }}$ e $\mathrm{N}_{\text {total }}$ seria a adsorção de nitrogênio inorgânico dissolvido, principalmente amônia, a partículas finas, entretanto, este fenômeno não parece ocorrer na área estudada devido à escassez da entrada de efluentes domésticos (Medeiros et al. 2011) e à natureza dos sedimentos arenosos, que por conta de sua porosidade contêm pouca amônia, produto gerado pela diagênese recente de nitrogênio orgânico.

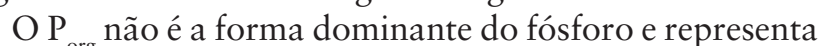
apenas $25 \%$ do total em sedimentos marinhos (Salomons \& Gerritse 1981). Na área estudada, esse percentual varia entre 33 e $45 \%$, sugerindo que a sua origem marinha é menos significativa que as fontes terrestres. A distribuição do Porg correlaciona-se à distribuição de sedimentos finos $(r=0,67 ; p<0,01 ; n=15)$, evidenciando a estreita relação entre os componentes da matéria orgânica. Dessa forma, as hipóteses sugeridas para explicar as relações entre $\mathrm{C}_{\text {org }}$ e $\mathrm{N}_{\text {total }}$ são válidas para $\mathrm{P}_{\text {org }}$. Observa-se que os sedimentos de manguezal se distinguem dos demais com maiores teores de $\mathrm{C}_{\text {org }}$ e $\mathrm{P}_{\text {org }}$.

As razões $\mathrm{C} / \mathrm{N}$ e $\mathrm{C} / \mathrm{P}$ indicam que a matéria orgânica sedimentar da área de estudo é pobre em nitrogênio e fósforo, característica de fonte terrestre (<20\%; Meyers 1994), o que é corroborado com correlações igualmente positivas e significativas entre as razões $\mathrm{C} / \mathrm{N}$ e $\mathrm{S} / \mathrm{V}$ nos sedimentos da área estudada. Keil et al. (1998) analisaram a relação entre a composição da matéria orgânica $(r=0,68 ; p=0,04 ; n=9)$ e a granulometria e relataram que detritos de plantas vasculares permanecem inalterados e em maior proporção na fração arenosa do sedimento.

Os valores de $\delta^{13} \mathrm{C}$ correlacionam significativamente com a razão $\mathrm{C} / \mathrm{N}(\mathrm{r}=-0,68 ; \mathrm{p}=0,01 ; \mathrm{n}=15)$, o que corrobora as tendências e hipóteses descritas anteriormente para explicar a distribuição da razão $\mathrm{C} / \mathrm{N}$ nos sedimentos estudados. Este padrão pode ser associado tanto à mudança de fonte de matéria orgânica quanto ao efeito de transformações diagenéticas. Entretanto, considerando que a decomposição da matéria orgânica ocorre preferencialmente na coluna de água e nos primeiros centímetros da coluna sedimentar (Colombo et al. 1996), é provável que as variações nos valores de $\delta^{13} \mathrm{C}$ e C/N são devidas à variação de fontes.

A interpretação das influências de fontes na composição da matéria orgânica sedimentar total é consolidada com a avaliação em conjunto dos dois parâmetros. De acordo com a Figura 2, aproximadamente metade das amostras são distribuídas entre as fontes vegetais analisadas, sendo a aninga (Montrichadia linifera) a espécie de maior influência. Segundo Santos (2001), a aninga é a vegetação dominante de mata ciliar em toda margem fluvial. A Rhizophora mangle contribui significativamente para a matéria orgânica sedimentar da estação S11, no manguezal. O sinal isotópico mais pesado, tanto do carbono quanto do nitrogênio, corresponde à macroalga Elodea sp., fonte significativa de matéria orgânica para a estação S2, onde esta alga ocorre em abundância. Entretanto, outra fonte não analisada está influenciando a composição da matéria orgânica sedimentar das amostras. Esta fonte provavelmente é o fitoplâncton, com baixa razão molar $\mathrm{C} / \mathrm{N}$ e assinatura isotópica mais pesada. Segundo Knoppers (dados não publicados), o valor de $\delta^{13} \mathrm{C}$ mediano do material fluvial em suspensão é aproximadamente -24,65\%o (-23,43 a $-27,10 \%$ ), medido $80 \mathrm{~km}$ rio adentro.

Os valores de $\delta^{15} \mathrm{~N}$ apresentam correlação significativa com os de $\delta^{13} \mathrm{C}(\mathrm{r}=0,52 ; \mathrm{p}<0,05 ; \mathrm{n}=15)$. Entretanto, nem 
todas as tendências e hipóteses descritas anteriormente para o $\delta^{13} \mathrm{C}$ e a razão $\mathrm{C} / \mathrm{N}$ se aplicam à distribuição do $\delta^{15} \mathrm{~N}$ nas amostras. $\mathrm{O}$ valor de $\delta^{15} \mathrm{~N}$ do material particulado em suspensão do RSF (Knoppers e Jennerjahn, dados não publicados) é aproximadamente $6,80 \%$ o $(6,43$ a $8,47 \%$ o), sendo típico de matéria orgânica de origem marinha (Matsuura \& Wada 1995). Os valores de $\delta^{15} \mathrm{~N}$ variando de 2 a $4 \%$ podem indicar tanto processos de denitrificação (Peterson \& Fry 1987) quanto simplesmente uma forte presença da Rhizophora mangle (3,05\%), influenciando, por exemplo, a estação $\mathrm{S} 11$. O $\delta^{15} \mathrm{~N}$ variando de 4 a $6 \%$ pode ser devido à Montrichardia linifera $\left(\delta^{15} \mathrm{~N}=5,48 \%\right.$ o), de ampla ocorrência nas margens fluviais.

A estação $\mathrm{CP}$ no pró-delta apresenta valor de razão $\mathrm{C} / \mathrm{N}$ e de ligninas comparáveis à estação S2 (Figura 2), coletadas aproximadamente $15 \mathrm{~km}$ à montante da foz. Nesta localidade, a macroalga Elodea sp. ocorre particularmente em abundância e apresenta o sinal mais pesado nos valores de $\delta^{13} \mathrm{C}$ e as menores concentrações de lignina entre as espécies vegetais analisadas. De fato, as macroalgas podem alcançar sinal mais pesado que -9\% (O’Leary 1988), características partilhadas com o fitplâncton. Entretanto, a pouca importância da fonte marinha fica evidente ao se constatar que a estação $\mathrm{CP}$ apresenta teores não negligenciáveis de ligninas (Figura 3). Além disso, as condições oligotróficas das águas costeiras já foram identificadas por vários autores (Ekau \& Knoppers 1999, Jennerjahn \& Ittekkot 1997, Jennerjahn et al. 1999, Jennerjahn \& Ittekkot 1999, Medeiros et al. 2007 e 2011, Knoppers et al. 2006) e reforçam a hipótese de influência insignificante de matéria orgânica marinha para esta estação costeira e o estuário.

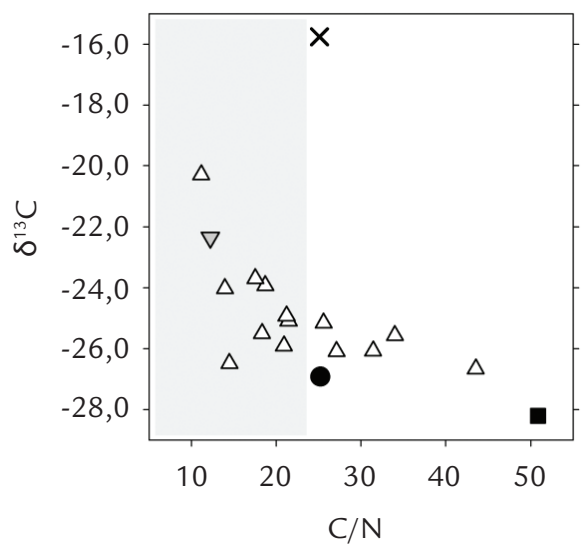

$\triangle$ surface sediments $\nabla C P \quad$ Rhizophora mangle

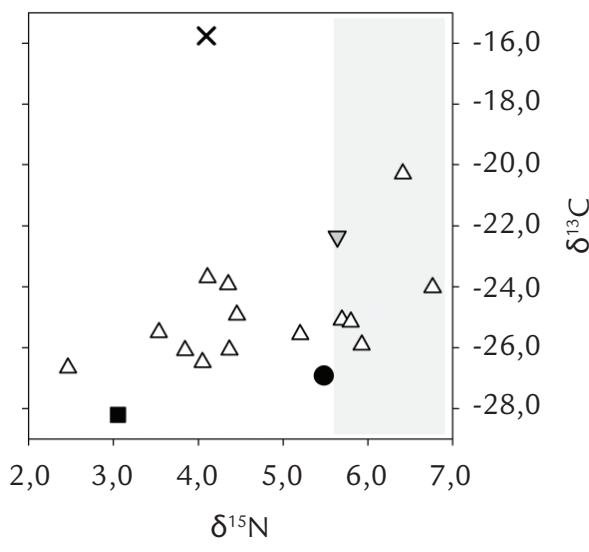

- Montrichardia linifera X Elodeasp.
Figura 2

Relações entre $\delta 13 C(\%)$, razão $C / N$ (molar) e $\delta 15 \mathrm{~N}$ (\%) dos sedimentos superficiais $(n=15)$ e das três espécies vegetais amostradas no delta-estuarino do Rio São Francisco, Nordeste do Brasil.

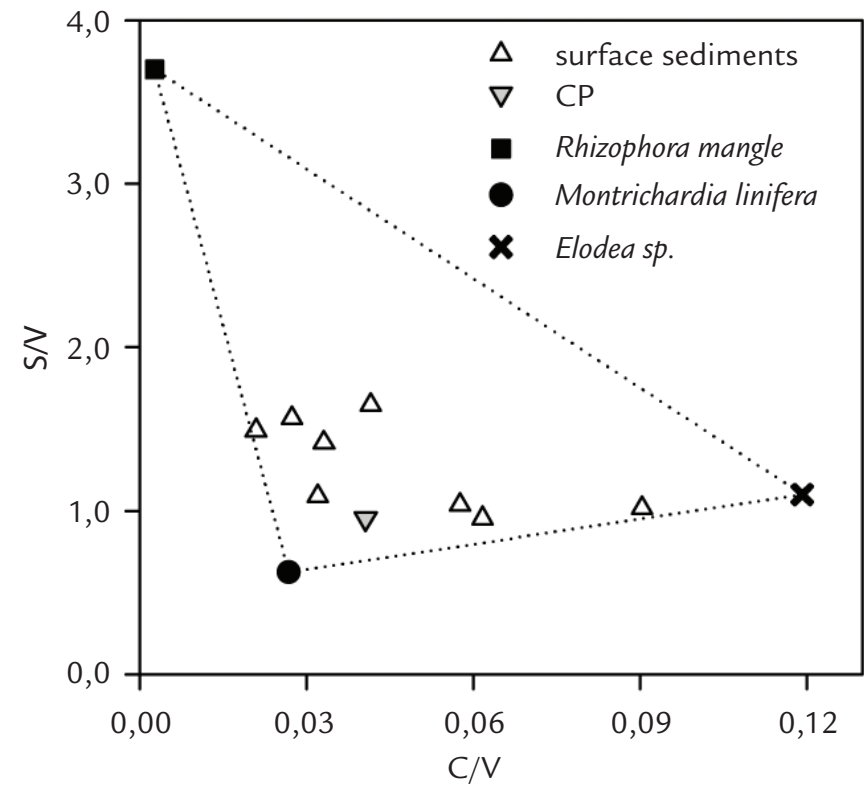

Figura 3

Relação entre razões Siringil/Vanilil (S/V) e Cinamil/Vanilil (C/V) dos sedimentos superficiais e das espécies vegetais amostradas no delta-estuarino do Rio São Francisco, Nordeste do Brasil. 


\subsection{Composição dos marcadores de ligninas}

Os compostos fenólicos da lignina analisados neste trabalho, comumente utilizados com sucesso na identificação da origem terrestre da matéria orgânica (Hedges \& Mann 1979, Hedges \& Parker 1976, Goñi \& Hedges 1992), suportam ainda mais as hipóteses levantadas sobre a forte influência terrestre nas amostras, identificadas pelas razões elementares e isotópicas. Nenhuma amostra sedimentar apresenta razão $\mathrm{C} / \mathrm{N}$ inferior a 10 e a maioria tem valor de $\delta^{13} \mathrm{C}$ superior a $-22 \%$ o (valores característicos de fonte marinha; Ingall \& Van Cappellen 1990, Meyers 1994, Meyers \& Ishiwatari 1993). Todas as amostras contêm V, S e C, indicando origem terrestre. Observa-se que quanto maior o conteúdo de lignina, maior a razão $\mathrm{C} / \mathrm{N}$ e menor o $\delta^{13} \mathrm{C}$, evidenciando a contribuição de plantas lenhosas.

A contribuição do material terrestre para a matéria orgânica sedimentar fica mais evidente ao se avaliar a relação das razões $\mathrm{S} / \mathrm{V}$ e $\mathrm{C} / \mathrm{V}$, que também permite discriminar a ocorrência de material lenhoso e foliar (Figura 3). A Montrichadia linifera apresenta baixos valores das razões acima mencionadas, o que a classifica como uma planta pouco lenhosa. Na região do gráfico de altos valores da razão $\mathrm{S} / \mathrm{V}$ (maior quantidade de material lenhoso) e baixos da razão C/V observam-se as angiospermas lenhosas, como a Rhizopora mangle (Farella et al. 2001, Goñi \& Hedges 1992, Hedges \& Mann 1979). Particularmente, as duas espécies não apresentam elevadas concentrações de C, o que é apenas observado na Elodea sp. As amostras sedimentares distribuem-se entre as espécies Rhizofora mangle, Montrichadia linifera (aninga) e Elodea sp. Segundo Santos (2001), a aninga é encontrada ao longo de toda a margem do canal central, a Rhizofora mangle ao longo de todo o canal secundário e a macroalga ao longo do leito fluvial central. A ampla distribuição da macroalga ao longo do rio é confirmada se considerarmos que, mesmo se esta é conhecidamente empobrecida em V e S (Hedges \& Mann 1979, Hedges et al. 1988, Farella et al. 2001), in- fluenciam significativamente as amostras sedimentares, que apresentam concentrações significativas dos produtos da oxidação da lignina do grupo P.

O aumento da forma ácida da vanilina em detrimento das formas aldeídica e cetônica é indicativo de degradação (Hedges et al. 1988, Hedges \& Prahl 1993). A razão (ac/al) $\mathrm{v}$ e (ac/al)s nas amostras analisadas não ultrapassa 0,4 (limite indicado pelos autores op.cite como indicativo de degradação), o que significa bom estágio de preservação dos sinais das fontes na matéria orgânica sedimentar e assegura confiabilidade às interpretações efetuadas.

Com o objetivo de integrar os dados discutidos e identificar possíveis padrões de distribuição da matéria orgânica sedimentar no estuário do RSF foi realizada a análise multiparamétrica de agrupamento. Da matriz de 15 amostras e 8 variáveis (sedimentos finos, C, N, P, C/N, C/P, $\delta 13 \mathrm{C}$ e $\delta 15 \mathrm{~N}$ ), evidenciaram-se dois grandes grupos: A e B. Os produtos de oxidação da lignina não foram considerados por não terem sido determinados em todas as amostras. As razões $\mathrm{C} / \mathrm{N}$, $\delta^{13} \mathrm{C}$ e teor de sedimentos finos foram fatores determinantes no agrupamento das estações. No grupo A estão reunidas as amostras que notoriamente apresentaram razões $\mathrm{C} / \mathrm{N}$ inferiores a 20 e o valor de $\delta^{13} \mathrm{C}$ mais pesado, sendo subdividido em grupo A2, amostra S2 com maior teor de finos, e grupo A1 (S1, S3, S9, S14 e CP) com menor teor de sedimentos finos. O grupo B foi formado pelas amostras com razões $\mathrm{C} / \mathrm{N}$ superiores a 20 e valor de $\delta^{13} \mathrm{C}$ mais leve, sendo subdividido em grupo B1 (S4, S5, S6, S8, S10, S12 e S13) com menor teor de sedimentos finos e grupo B2 com maior teor de sedimentos finos (S7 e S11) (Figura 4). Os dois grupos 100\% distintos representam a significância equivalente das fontes de matéria orgânica para os sedimentos do estuário, que são plantas superiores terrestres e algas. A granulometria tem menor significância na distribuição da matéria orgânica sedimentar.

Ward's method

City-block (Manhattan) distances
Figura 4

Dendograma da matriz de 15 amostras e 8 variáveis (sedimentos finos, $C, N, P, C / N$, $\mathrm{C} / \mathrm{P}, \delta 13 \mathrm{C}$ e $\delta 15 \mathrm{~N}$ ) determinadas nos sedimentos superficiais do delta-estuarino do

Rio São Francisco, Nordeste do Brasil.

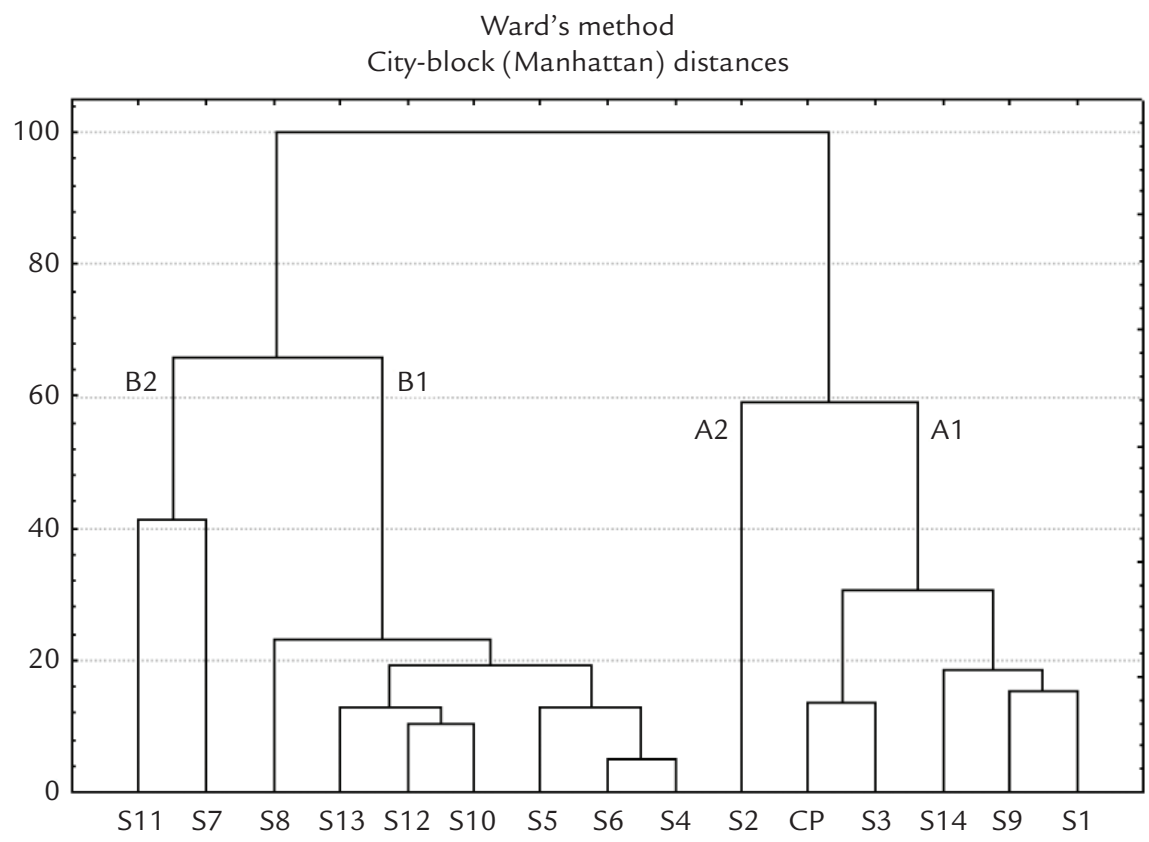




\section{CONCLUSÃO}

Várias consequências decorreram da regularização da vazão fluvial após a construção da barragem do Xingó, como redução do aporte de sedimentos e de nutrientes para o estuário e para a zona costeira, fortalecendo a erosão e diminuindo a produtividade biológica do sistema. A erosão das margens é um processo desencadeado pela queda acentuada do nível de água fluvial, alterando o raio hidráulico e desestabilizando pacotes sedimentares marginais ao longo de quase todo o percurso fluvial no Baixo São Francisco. O menor aporte de água fluvial também magnificou o efeito de ressuspensão sedimentar das ondas, alterando o equilíbrio dinâmico-sedimentar na zona costeira. Esses processos ocorrem simultaneamente na área estudada, porém atuam espacialmente em diferentes intensidades. A distribuição espacial da granulometria e dos demais parâmetros analisados reflete claramente esse cenário e distingue as localidades mais erosivas das outras mais deposicionais, características de regiões mais enclausuradas, como a região central do manguezal e a pequena enseada fluvial.

Essa acentuada hidrodinâmica, identificada pelo teor de sedimentos finos predominantemente inferior a $70 \%$, justifica as baixas concentrações de matéria orgânica encon-

\section{AGRADECIMENTOS}

A primeira autora recebeu uma bolsa de doutorado do Conselho Nacional de Desenvolvimento Científico e Tecnológico (CNPq) e de estágio de doutoramento no exterior Programa de Doutorado no País com Estágio no Exterior (PDEE) da Coordenação de Aperfeiçoamento de Pessoal de Nível Superior (CAPES). O suporte tradas, características de ambiente oligotrófico, classificação também observada na coluna de água por outros trabalhos desenvolvidos na mesma área. As principais fontes de matéria orgânica para os sedimentos do estuário do RSF são a fonte flúvio-terrestre e as espécies vegetais Montrichadia linifera (aninga), Rhizophora mangle e a macroalga Elodea $s p$., contribuições significativas claramente identificadas pelas razões $\mathrm{C} / \mathrm{N}$, isotópicas e dos produtos de oxidação da lignina. A surpreendente importância da macroalga como fonte de matéria orgânica é também consequência das drásticas reduções de vazão e material particulado em suspensão às quais o RSF foi submetido.

A discussão aqui apresentada sobre sinal isotópico de carbono e nitrogênio em amostras sedimentares acentua a necessidade de se determinar a assinatura elementar, isotópica e molecular de fontes vegetais tropicais, e, assim, estabelecer novos índices apropriados, pois a enorme biodiversidade, as melhores condições favoráveis de produção ao longo de todo o ano e a menor disponibilidade de nutrientes (principalmente em águas costeiras) em relação às zonas temperadas se traduzem em assinaturas muito diferentes das observadas em outras latitudes.

financeiro do $\mathrm{CNPq}$ foi obtido da bolsa de pesquisa concedida a Bastiaan Knoppers (Proc. Nr. 300772/2004-1) e do Projeto Instituto do Milênio-Estuaries (Proc. Nr. 420050/2005-1) e CNPq-Instituto Nacional de Ciência e Tecnologia de Transferência de Materiais Continente-Oceano (INCT-TMOcean) Proc. Nr. 573601/2008-9

\section{REFERÊNCIAS}

ANA/GERCO/PNUMA/OEA. 2003. Projeto de Gerenciamento Integrado das Atividades Desenvolvidas em Terra na Bacia do São Francisco. Disponível em: http://www.ana. gov.br/gefsf. Acessado em 5 maio 2005.

Aspila K.I., Argemain H., Chau A.S.Y. 1976. A semi-automated method for the determination of inorganic, organic and total phosphorus in sediments. Analyst, 101:187-197.

Baptista Neto J.A., Smith B.J., McAllister J.J. 2000. Heavy metal concentrations in surface sediments in a nearshore environment, Jurujuba Sound, Southeast Brazil. Environ Pollut, 109:1-9.

Bernardes L.M.C. 1951. Notas sobre o clima da bacia do Rio São Francisco. Rev Bras Geogr, 13:473-489.

Bessa M.F. \& Paredes J.F. 1990. Transporte do carbono e do nitrogênio orgânico e inorgânico dissolvidos pelo Rio São Francisco, Brasil, durante um ano hidrológico (1984-1985). Geoch Bras, 4:17-31.

Billen G., Lancelot C., Meybeck M.1991. N, P and Si retention along the aquatic continuum from land to ocean. In: Mantoura R.F.C., Martin J.M., Wollast R. (eds.). Ocean Margin Processes in Global Change. John Wiley \& Sons, New York, p. 19-44.

Bittencourt A.C.S.P., Dominguez J.M.L., Fontes L.C.S., Sousa D.L., Silva I.R., Silva F.R. 2007. Wave refraction, river damming, and episodes of severe shoreline erosion: The Sao Francisco River Mouth, Northeastern Brazil. JCR, 23:930-938.

Burone L., Muniz P., Pires-Vanin A.M., Rodrigues M. 2003. Spatial distribution of organic matter in the surface sediments of Ubatuba Bay (Southeastern - Brazil). An Acad Brasil Ciênc, 75:77-90. 
CODEVASF - COMPANHIA DE DESENVOLVIMENTO DO VALE DO RIO SÃO FRANCISCO. 1975. II Plano Nacional de Desenvolvimento: programa de ação do governo para o Vale do São Francisco, 1975-1979. CODEVASF, Brasília, 184 p.

Colombo J.C., Silverberg N., Gearing J.N. 1996. Biogeochemistry of organic matter in the Laurentian Through, II. Bulk composition of sediments and relative reactivity of major components during early diagenesis. Mar Chem., 51:295-314.

Cowie G.L. \& Hedges J.I. 1992. Sources and reactivity of amino acids in a coastal marine environment. Limnol Oceanogr, 37:703-724.

Dominguez J.M. 1996. The Sao Francisco strandplain: a paradigm for wave-dominated deltas? In: De Batist M. \& Jacobs P.. (eds.) Geology of Siliciclastic Shelf Seas. Geological Society, London, p. 217-231.

Ekau W. \& Knoppers B. 1999. An introduction to the pelagic system of the North-East and East Brazilian shelf. Arch Fish Mar Res, 47:113-132.

Farella N., Lucotte P., Louchouarn P., Roulet M. 2001. Deforestation modifying terrestrial organic transport in the Rio Tapajos, Brazilian Amazon. Organic Geochemistry, 32:1443-1458.

Folk R.L. \& Ward W.C. 1957. Brazos River bar: a study in the significance of grain size parameters. J Sediment Petrol., 27:3-26.

Goñi M.A. \& Hedges J.I. 1992. Lignin dimmers: structures, distribution, and potential geochemical applications. Geoch Cosm Act, 56:4025-4043.

Hedges J.I. \& Ertel J.R. 1982. Characterization of lignin by capillary gas chromatography of cupric oxide oxidation products. Anal Chem, 54:174-178.

Hedges J.I., Clark W.A., Quay P.D., Richey J.E., Devol A.H., Santos U. 1986. Composition and fluxes of particulate organic material in the Amazon River. Limnol Oceanograph, 31:717-738.

Hedges J.I., Clark W.A., Cowie G.L.1988. Organic matter sources to the water column and surficial sediments of a marine bay. Limnol Oceanogr, 33:1116-1136.

Hedges J.I. \& Mann D.C. 1979. The characterization of plant tissues by their lignin oxidation products. Geoch Cosm Act, 43:1803-1807.

Hedges J.I. \& Parker P.L. 1976. Land-derived organic matter in surface sediments from the Gulf of Mexico. Geoch Cosm Act, 40:1019-1029.

Hedges J.I. \& Prahl F.G. 1993. Early diagenesis: consequences for applications of molecular biomarkers. In: Engel M. \& Mackro S.A. (eds.) Organic geochemistry: principles and applications. Plenum Press, New York, p. 237-253.

Ingall E.D. \& Van Cappellen P. 1990. Relation between sedimentation rate and burial of organic phosphorus and organic carbon in marine sediments. Geoch Cosm Act, 54:373-386.

Ittekkot V. \& Zhang S. 1990. Pattern of particulate nitrogen transport in world rivers. Global Biogeochem Cycles, 3:383-391.

Jennerjahn T.C. \& Ittekkot V. 1997. Organic matter in sediments in the mangrove areas and adjacent continental margins of Brazil: I. Amino acids and hexosamines. Oceanol Acta, 20:359-369.

Jennerjahn T.C. \& Ittekkot V. 1999. Changes in organic matter from surface waters to continental slope sediments off the São Francisco River, eastern Brazil. Marine Geology, 161:129-140.

Jennerjahn T.C. \& Ittekkot V. 2002. Relevance of mangroves for the production and deposition of organic matter along tropical continental margins. Naturwissenschaften, 89:23-30.

Jennerjahn T.C., Ittekkot V., Carvalho C.E.V., Ovalle A.R.C., Rezende C.E., Erlenkeuser, H. 1999. Temporal variability of amino acid, hexosamine, and carbohydrate fluxes on the eastern Brazilian continental margin related to discharge of the São Francisco River, Brazil. Geo-Marine Letters, 19:202-208.

Keil R.G., Tsamakis E., Giddings J.C., Hedges J.I. 1998. Biochemical distributions (amino acids, neutral sugars, and lignin phenols) among size-classes of modern marine sediments from the Washington coast. Geoch Cosm Act, 62:1347-1364.

Knoppers B., Medeiros P.R.P., Souza W.F.L., Jennerjahn T. 2006. The São Francisco Estuary, Brazil. Hdb Env Chem, 5:51-70.

Koroleff F. 1976. Determination of phosphorus. In: Grasshoff K. (ed.) Methods of seawater analysis. Verlag Chemie, Weinheim/New York, p. 134-136. 
Kögel I. \& Bochter R. 1985. Characterization of lignin in forest humus layers by highperformance liquid chromatography of cupric oxide oxidation products. Soil Biol Biochem, 17:637-640.

Oliveira A.M. 2003. Estudo hidrodinâmico-sedimentológico do Baixo São Francisco, estuário e zona costeira adjacente (AL/SE). Relatório Final. In: ANA/GEF/PNUMA/ OEA. Projeto de Gerenciamento Integrado das Atividades Desenvolvidas em Terra na Bacia do São Francisco. Universidade Federal de Alagoas, Alagoas, 33 p.

Mantoura R.F.C., Martin J. M., Wollast R. (eds.) 1991. Ocean margin processes in global change. John Wiley \& Sons, Chichester, 469 p.

Marques M., da Costa M.F., Mayorga M.I., Pinheiro P.R. 2004. Water environments: anthropogenic pressures and ecosystem changes in the Atlantic drainage basins of Brazil. Ambio, 33:68-77.

Matsuura Y. \& Wada E. 1995. Carbon and nitrogen stable isotope ratios in marine organic matters of the coastal ecosystem in Ubatuba, southern Brazil. Cienc Cult, 46:141-146.

Mauriel M.C.O. 2004. Características biogeoquímicas do canal do funil, estuário do Rio São Francisco, SE, Brasil. Dissertação de Mestrado, Instituto de Química, Universidade Federal Fluminense, $107 \mathrm{p}$.

Medeiros P.R.P., Knoppers B.A., dos Santos Jr R.C., Souza W.F.L. 2007. Aporte fluvial e dispersão de matéria particulada em suspensão na zona costeira do Rio São Francisco (SE/AL). Geoch Bras, 2:209-228.

Medeiros P.R.P., Knoppers B.A., Souze W.F.L., Oliveira E.N. 2011. Aporte de material em suspensão no baixo rio São Francisco (SE/AL) em diferentes condições hidrológicas. Braz J Aquat Sci Technol, 15:42-53.

Meyers P.A. \& Ishiwatari R. 1993. Lacustrine organic geochemistry - an overview of indicators of organic matter sources and diagenesis in lake sediments. Org Geoch, 20:867-900.

Meyers P.A. 1994. Preservation of source identification of sedimentary organic matter during and after deposition. Chem Geol, 144:289-302.

Miltner A. \& Emeis K.C. 2001. Terrestrial organic matter in surface sediments of the Baltic Sea, NW Europe, as determined by CuO oxidation. Geochim Cosmochim Acta, 65:1285-1299.

Milligan G.W.1988. A study of standardization of variables in cluster analysis. Journal of Classification, 5:181-204.

O'Leary M.H. 1988. Carbon isotopes in photosynthesis. Bioscience, 38:328-336.

Oliveira E.N., Knoppers B.A., Lorenzzetti J.A., Medeiros P.R.P., Carneiro M.E., Souza W.F.L. 2012. A satellite view of riverine turbidity plumes from the NE-E Brazilian coastal zone. Braz J Oceanogr, 60:283-298.

Peterson B.J. \& Fry B. 1987. Stable isotopes in ecosystems studies. Annual Review of Ecological and Systematics, 18:293-320.

Pritchard D.W. 1952. Salinity distribution and circulation in the Chesapeake Bay estuarine system. J Mar Res, 11:106-123.

Ruttemberg K.C. \& Goñi M.A. 1997. Phosphorus distribution, C:N:P ratios, and $\delta 13 \mathrm{Coc}$ in arctic, temperate, and tropical coastal sediments: tools for characterizing bulk sedimentary organic matter. Marine Geology, 139:123-145.

Sabadini-Santos E., Knoppers B.A., Oliveira E.P., Leipe T., Santelli R.E. 2009. Regional geochemical baselines for sedimentary metals of the tropical São Francisco estuary, NE-Brazil. Mar Pollut Bull, 58:601-606.

Salomons W. \& Gerritse R.G. 1981. Some observations on the occurrence of phosphorus in recent sediments from western Europe. Sci Total Environ, 17:37-49.

Santos L.G.C. 2001. Diagnóstico dos remanescentes de mata ciliar no Baixo São Francisco Sergipano afetado pela erosão marginal e a compreensão dos ribeirinhos sobre a degradação desta vegetação. Dissertação de Mestrado, Universidade Federal do Sergipe, 111 p.

Tundisi J.G., Rocha O., Matsumura-Tundisi T., Braga B. 1998. Reservoir Management in South America. Int J Water Resour D, 14:141-155.

Ver L.M.B., Mackenzie F.T., Lerman A. 1999. Carbon cycle in the coastal zone: effects of global pertubations and change in the past three centuries. Chemical Geology, 159:283-304.

Zar, J.H. 1982. Biostatistical analysis. 2 ed. Prentice-Hall Inc., New Jersey, 718 p. 